\title{
Effect of Aging on Auto-Antibodies to Wounded Tissues
}

\author{
Naomi Nishio ${ }^{1}$, Sachiko Ito $^{1}$, Haruhiko Suzuki ${ }^{1}$, Yayoi Okawa ${ }^{2}$ and Ken-ichi Isobe ${ }^{1, *}$ \\ ${ }^{I}$ Department of Immunology, Nagoya University Graduate School of Medicine, 65 Turumai-cho, Showa-ku, Nagoya, \\ Aichi, 466-8520, Japan \\ ${ }^{2}$ Department of Functioning Activation, National Institute for Longevity Sciences, National Center for Geriatrics \\ Gerontology, Aichi, 463-0003, Japan
}

\begin{abstract}
Wound healing succeeds tissue destruction. We hypothesized that antibodies might bind to wounded tissues, which would facilitate the engulfment of damaged tissues by macrophages. We detected the autoantibodies in sera of different ages of mice, which bind to wounded tissues. We detected IgG1 binding to wounded tissues by using FITClabeled anti-IgG1 in C57BL/6 mice. These bands were highest in 4 months old C57BL/6 mice. The sera taken from 2 months and 20 months old mice also bound to wounded tissues, although the bands were weaker than those of 4 months old mice. We also examined the autoreactive IgM binding to wounded tissues. We could detect relatively strong bands even in $2 \mathrm{M}$ old mice. The pattern of these bands was changed by advancing age. Intensity of IgM bands was not decreased by advancing age. The splenectomy reduced the intensity of IgG1 bands especially in 4 months old mice. Although we observed the slight delay of wound repair by splenectomy in $2 \mathrm{M}$ and $20 \mathrm{M}$ old mice, we observed that wound repair was strongly delayed in $4 \mathrm{M}$ old mice. Serum from any age of mice enhanced the macrophage phagocytosis by opsonization. Serum taken from splenectomized mice decreased the opsonizing capacity only at $4 \mathrm{M}$.
\end{abstract}

Keywords: Aging, IgG1, wound healing, splenectomy.

\section{INTRODUCTION}

One of the most important clinical problems in caring for elderly patients is treatment of pressure ulcers. Pressure ulcers are caused by compression, friction, infection and/or malnutrition. The ability to heal pressure ulcers declines with age [1]. To understand the mechanism underlying the impaired ability of older patients to repair pressure ulcers, we established studies to analyze wound healing in aged mice. The wound repair process is a highly ordered sequence of events that encompasses hemostasis, inflammatory cell infiltration, and tissue re-growth and remodeling [2]. Initially, neutrophils accumulate at sites of injury, followed by a large influx of macrophages. The function of granulocytes and macrophages in wound healing and inflammation has been extensively studied [3-5]. Neutrophils have been shown to prevent wound repair processes in neutrophil depletion studies [6]. These studies were done using young mice. However, we have shown in aged mice that the depletion of neutrophils by anti-Gr-1 antibody dramatically delayed wound healing [7].

The main function of macrophages and neutrophils is phagocytosis of pathogens and cellular debris. These cells phagocytize apoptotic and necrotic cells directly through use

*Address correspondence to this author at the Department of Immunology, Nagoya University Graduate School of Medicine, Nagoya University Graduate School of Medicine, 65 Tsurumai-cho, Showa-ku, Nagoya, Aichi, 466-8520, Japan; Tel: +81-52-744-2135; Fax: +81-52-744-2972;

E-mail: kisobe@med.nagoya-u.ac.jp of their phagocytic receptors [8]. Clearance of damaged tissue and debris proceeds more efficiently when autoantibodies attach to the wounded tissues (opsonization). There are several reports which describe the effects of T or B cells in wound healing, with the exception of $\gamma \delta \mathrm{T}$ cells $[9$, 10]. In aseptic wound healing, very few $T$ or $B$ cells migrate to the wound site. However, immunoglobulins secreted by B cells can reach the wound sites. Recently, in order to examine the effects of immunoglobulin, we performed splenectomy in a mouse model and found that it greatly delayed wound healing by reducing the amount of IgG1 binding to the damaged tissues [11]. To better understand the role of autoantibodies in the repair of pressure ulcers in older patients, we examined $\operatorname{IgM}$ and $\operatorname{IgG1}$ autoantibodies bound to wounded tissues from both young and aged mice. Further, we examined whether wound healing was accelerated by these autoantibodies.

\section{MATERIALS AND METHODS}

\section{Mice and Splenectomy}

Two months (2M) old C57BL/6 female mice were purchased from SLC Japan and maintained throughout their lifespan in the Animal Research Facility at the Nagoya University Graduate School of Medicine under specific pathogen-free conditions and used according to institutional guidelines. Mice (4M, 6M, 12M, 17M, 20M, 26M old) were also maintained there. The aged mice were free of cancer and other diseases. In splenectomy experiment, mice of different ages $(2 \mathrm{M}, 4 \mathrm{M}, 20 \mathrm{M})$ were anesthetized and 
subjected to either a sham operation or splenectomy. One week later, punch biopsies were performed on the backs of these mice.

\section{Punch Biopsy Wounding and Macroscopic Examination}

After shaving and extensive cleaning with $70 \%$ ethanol, the dorsal skin was picked up at the midline and two layers of skin were punched through with a sterile disposable biopsy punch (diameter $3 \mathrm{~mm}$; Kai Industries). This procedure generated two excision full-thickness wounds with one on each side of the midline. The same procedure was repeated four times, generating eight wounds on each animal. Each wound site was digitally photographed at the indicated time intervals, and wound areas were determined on photographs using Photoshop (version 7.0; Adobe Systems) and the areas were calculated with Excel. Changes in the areas of wounded sites were expressed as the proportion of the initial wound areas. For Western blotting, wounds and their surrounding areas (including the scab and epithelial margins) were cut for further analyses with a sterile disposable biopsy punch with a diameter of $6 \mathrm{~mm}$ (Kai Industries) at the indicated time points.

\section{Auto-Antibody Detection by Western Blotting}

Tissues were homogenized in buffer $(50 \mathrm{mM}$ Tris- $\mathrm{HCl}$ $\mathrm{pH} 6.8,2 \%$ SDS, 2 mM EDTA-2Na), and then lysed in sample buffer (50 mM Tris-HCl pH 6.8, 2\% SDS, 2.5\% glycerol, $5 \%$ 2-mercaptoethanol, and $0.05 \%$ bromophenol blue). Total protein $(1 \mathrm{mg} / \mathrm{mL})$ was resolved by SDS-PAGE and then transferred to PVDF membranes (Millipore). Blotted membranes were reacted with $1 / 100$ diluted serum. Then, membranes were reacted with goat anti-mouse IgG1 secondary antibodies or anti-mouse IgM secondary antibodies (Santa Cruz). Bound antibodies were detected with an enhanced chemiluminescence (ECL) system (Amersham Pharmacia). The intensity of bands in Western blots was determined by image analysis software (Lumi Vision Analyzer 400 product by AISHIN).

\section{Phagocytosis by Macrophages}

Three days after injection of $1 \mathrm{~mL}$ of $3 \%$ thioglycollate medium, peritoneal macrophages were collected from groups of three mice and were isolated by adherence of the cells to tissue culture plates overnight at $37^{\circ} \mathrm{C}$ in $5 \% \mathrm{CO}_{2}$. Murine peritoneal neutrophils were collected as described before by injecting $0.5 \mathrm{mg}$ zymosan intraperitoneally [7]. Neutrophils were treated with CFSE, then damaged by $10 \mathrm{sec}$ exposure to $100^{\circ} \mathrm{C}$ heat. Macrophages $\left(5 \times 10^{5}\right)$ and targets (damaged neutrophils) were plated at a ratio of one macrophage to one target cell. The mixture was incubated at $37^{\circ} \mathrm{C}$ in $5 \% \mathrm{CO}_{2}$ for $1 \mathrm{~h}$ and washed with PBS to remove unincorporated target cells. They were detached by trypsin/EDTA treatment. Cells were assessed on a flow cytometer (FACSCalibur, Becton Dickinson), and analyzed using FlowJo software (Tomy Digital Biology).

\section{RESULTS}

\section{IgG1 and IgM Binding to Wounded Tissues: Age Differences}

Although we detected distinct bands in $2 \mathrm{M}$ old mice, the intensities and varieties of bands from $2 \mathrm{M}$ old mice were low. The intensity of the bands was greatly increased at $4 \mathrm{M}$. Although the pattern of these bands changed with advancing age, we could detect specific bands during aging. In previous paper, we specified some of the bands by mass spectroscopy [11]. Because each bands in this paper may contain another proteins, we tentatively named the bands by molecular weight. We measured the intensity of each band observed in the gels. Many bands gradually increased with advancing age, peaking around $17 \mathrm{M}$ old $(220 \mathrm{~K}, 150 \mathrm{~K}$ and $100 \mathrm{~K})$ or $12 \mathrm{M}$ old $(43 \mathrm{~K}$ and $75 \mathrm{~K})$. Almost all bands decreased at $20 \mathrm{M}$ of age (Fig. 1). When we examined auto-reactive IgM binding to damaged tissues, we detected relatively strong bands, even in $2 \mathrm{M}$ old mice. The pattern of these bands changed during aging. The intensity of $\operatorname{IgM}$ bands did not decrease over time (Fig. 2).

\section{Age-Dependent Reduction in the Binding of IgG1 Anti- bodies to Damaged Tissues after Splenectomy}

Because the IgG1 antibody binding pattern to wounded tissues changed with advancing age, we examined the effects of splenectomy. At $4 \mathrm{M}$ of age, the IgG1 bound to wounded tissues was dramatically decreased by splenectomy. However, the IgG1 bound to damaged tissues in $20 \mathrm{M}$ old C57BL/6 mice decreased less than that observed with $4 \mathrm{M}$ old mice following splenectomy (Fig. 3A). By gel intensity analysis, we found that almost all bands $(220 \mathrm{~K}, 150 \mathrm{~K}, 100 \mathrm{~K}$, $75 \mathrm{~K}$ and $43 \mathrm{~K}$ ) were greatly decreased by splenectomy at $4 \mathrm{M}$. These bands were decreased in $20 \mathrm{M}$ old mice. However, in splenectomized $2 \mathrm{M}$ old mice, we observed only a slight decrease of 220K, 150K, 100K, 75K (Fig. 3B).

\section{Delayed Wound Repair in Splenectomized Mice is Age- Dependent}

Two-month, $4 \mathrm{M}$ or $20 \mathrm{M}$ old C57BL/6 mice were splenectomized or given sham operations. At one week postoperation, we performed punch biopsies on the backs of these mice. Although we observed a slight delay of wound repair following splenectomy in $2 \mathrm{M}$ and $20 \mathrm{M}$ old mice, we observed that wound repair was strongly delayed in $4 \mathrm{M}$ old mice (Fig. 4).

\section{Effects of Aging on Opsonization}

Antibodies to the damaged tissues might facilitate phagocytosis by opsonization of the damaged tissues. Therefore, we asked whether serum antibodies might enhance phagocytosis by macrophages. We used neutrophils as damaged cells in this experiment. We found that the addition of serum to heat-damaged neutrophils facilitated phagocytosis by macrophages. The extent of phagocytosis without serum was $13.3 \%$. The percentages of phagocytosis with serum from $2 \mathrm{M}$ old, $4 \mathrm{M}$ old and $20 \mathrm{M}$ old mice were $36.0 \%, 45.2 \%$ and $39.7 \%$, respectively. These results indicate that opsonization by serum greatly enhances wound healing at all ages. Only serum taken from splenectomized mice at $4 \mathrm{M}$ decreased the opsonizimg capacity. The opsonizing capacity of the serum taken from splenectomized mice at $2 \mathrm{M}$ or $20 \mathrm{M}$ old did not significantly differ from that of sham-operated mice (Fig. 5).

\section{DISCUSSION}

Here we have shown that the pattern of $\operatorname{IgG} 1$ antibodies targeted against damaged tissues changed with advancing 
(A)

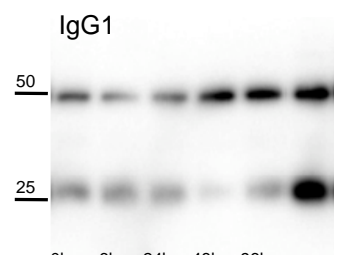

$2 \mathrm{M}$



Oh, $6 h, 24 h, 48 h, 96 h$, serum

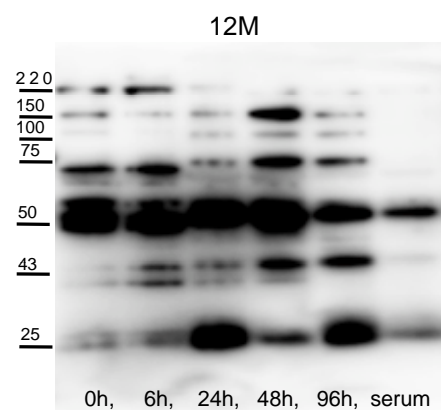

(B)

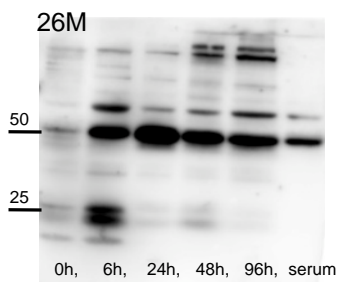

$4 \mathrm{M}$

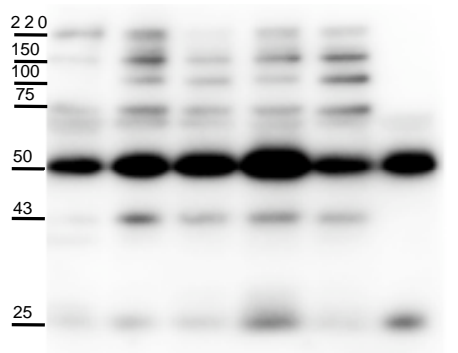

Oh, $6 h, \quad 24 h, 48 h, 96 h$, serum

$17 \mathrm{M}$

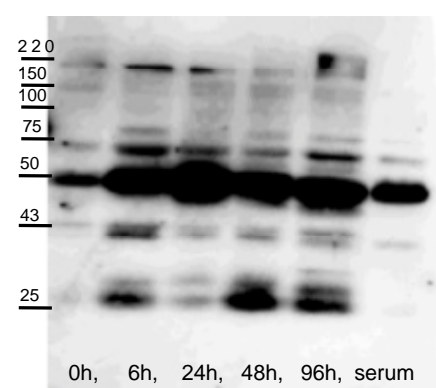

$6 \mathrm{M}$

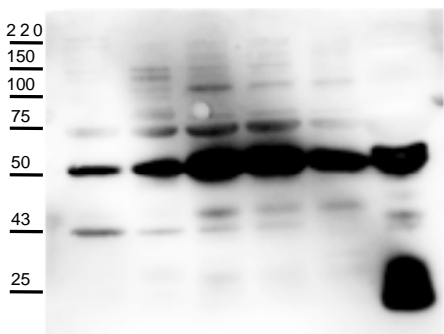

Oh, $6 h, 24 h, 48 h, 96 h$, serum

$20 \mathrm{M}$

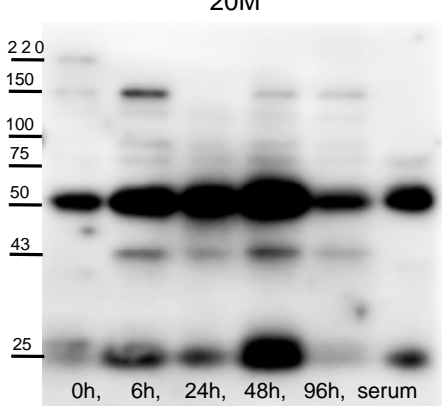


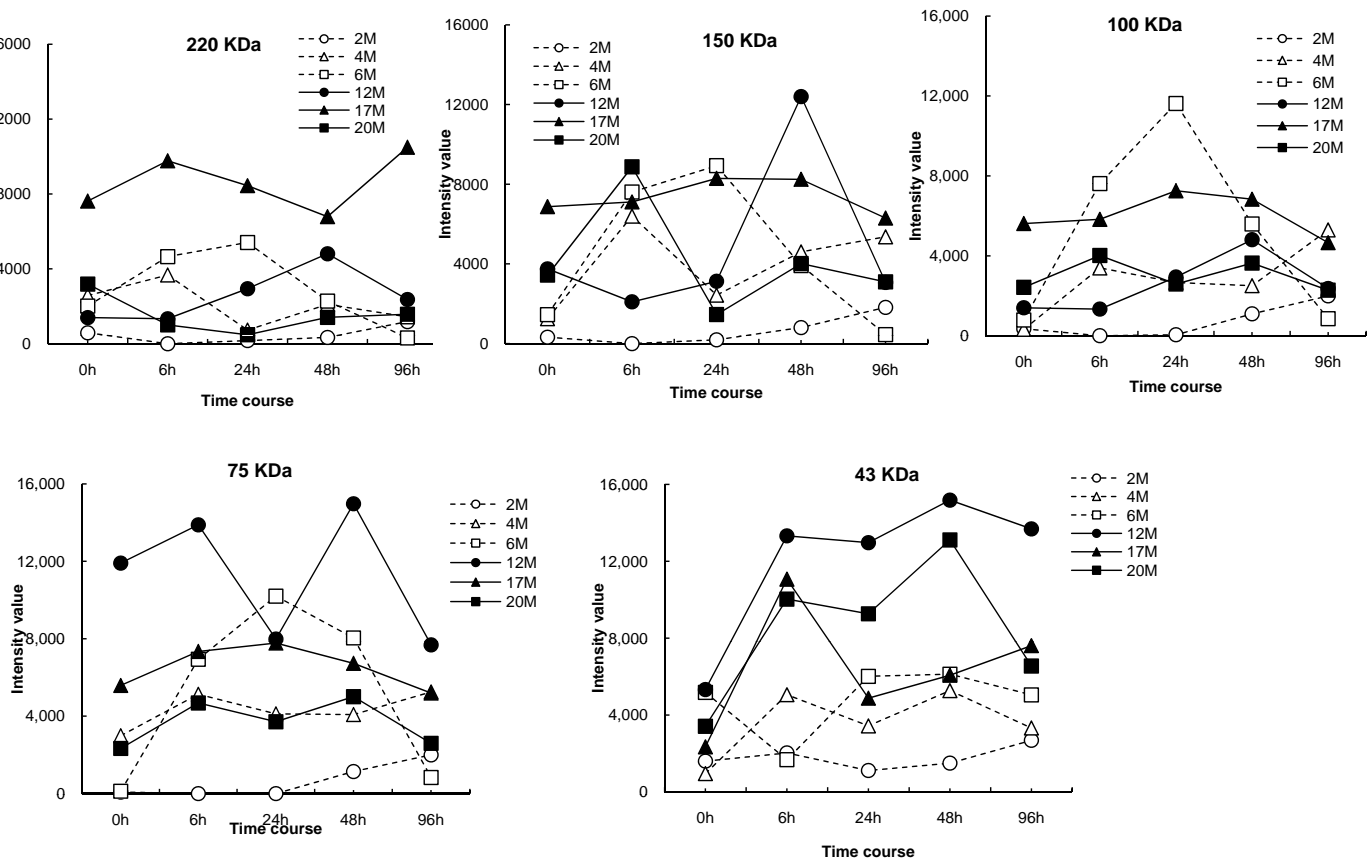

Fig. (1). Age-related changes of IgG1 auto-antibodies bound to damaged skin.

Damaged tissues were taken from $2 \mathrm{M}$ old C57BL/6 mice at $0 \mathrm{~h}$, and $6 \mathrm{~h}$ to $96 \mathrm{~h}$ after punch biopsy. Tissue lysates/homogenates were subjected to SDS PAGE and transferred to PVDF membranes. Sera (diluted 1:100) taken from 2M, 4M, 6M, 12M, 17M, 20M or 26 M old mice were reacted with membranes as a source of primary antibodies and then the membranes were reacted with anti-mouse IgG1 secondary antibodies. A. Bound antibodies were detected by the enhanced chemiluminescence (ECL) system. Representative results are shown for the sera taken from five mice at each age. Lines indicate the possible bands for quantitative analysis. B. Determination of band intensity by image analysis software. Intensity of each band was determined after subtracting background intensity around the band. 


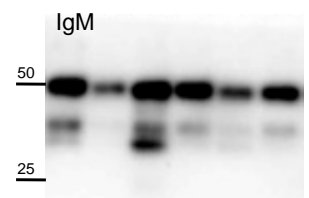

Oh, $6 h, 24 h, 48 h, 96 h$, serum

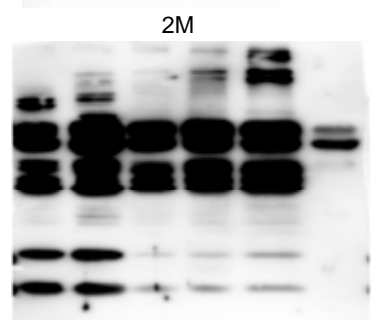

Oh, $6 h, 24 h, 48 h, 96 h$, serum

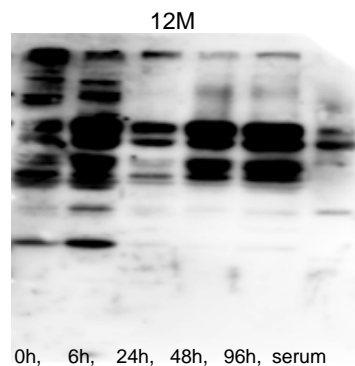

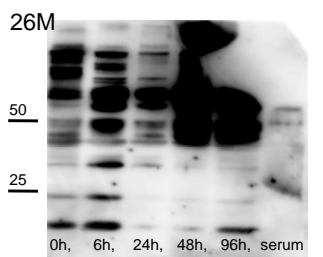

$4 \mathrm{M}$

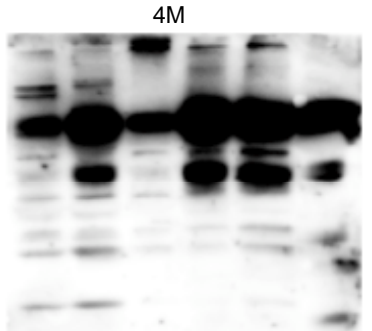

Oh, $6 h, \quad 24 h, 48 h, 96 h$, serum



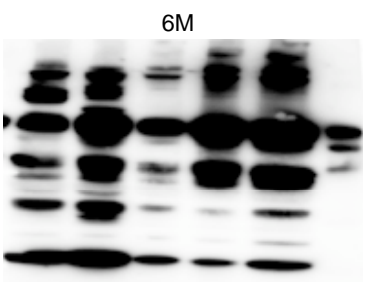

Oh, $6 \mathrm{~h}, \quad 24 \mathrm{~h}, 48 \mathrm{~h}, 96 \mathrm{~h}$, serum

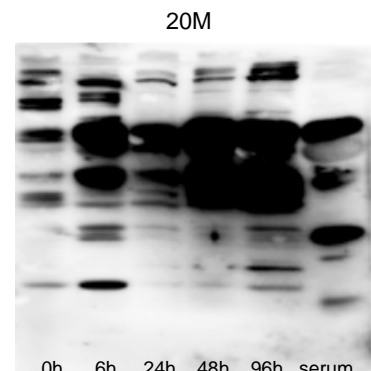

Fig. (2). Age-related changes of $\operatorname{IgM}$ auto-antibodies bound to damaged skin.

Damaged tissues were taken from 2M old C57BL/6 mice at $0 \mathrm{~h}$, and $6 \mathrm{~h}$ to $96 \mathrm{~h}$ after punch biopsy. Tissue lysates/homogenates were subjected to SDS PAGE and transferred to PVDF membrane. Sera (diluted 1:100) taken from 2M, 4M, 6M, 12M, 17M, 20M or 26 M old mice were reacted with membranes as a primary antibody and then membranes were reacted with anti-mouse IgM secondary antibodies. Bound antibodies were detected by the enhanced chemiluminescence (ECL) system. Representative results are shown for sera taken from five mice at each age.

(A)

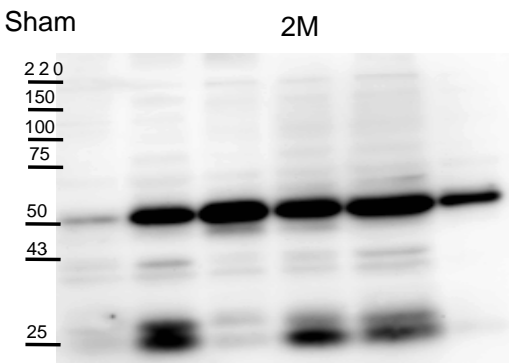

Oh, 6h, 24h, 48h, 96h, serum

Splenectomy

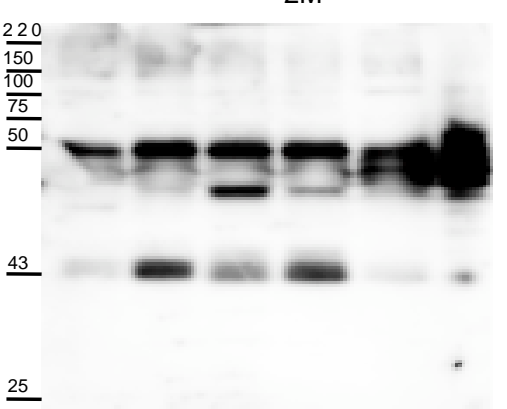

$4 \mathrm{M}$

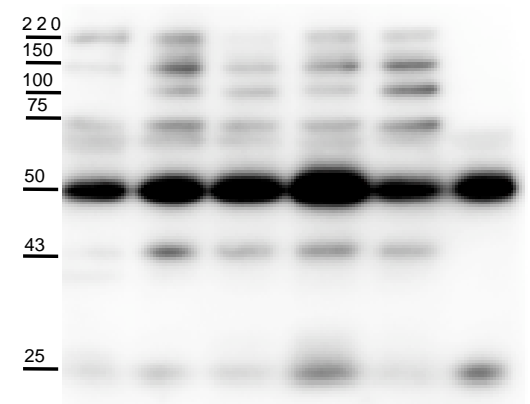

Oh, 6h, 24h, 48h, 96h, serum

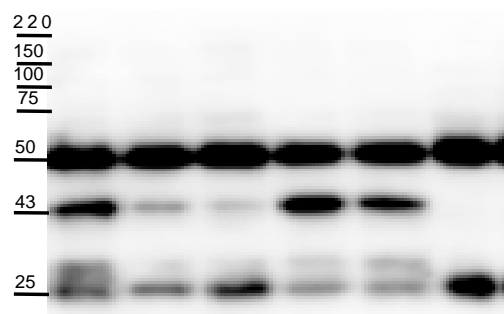

\section{.}

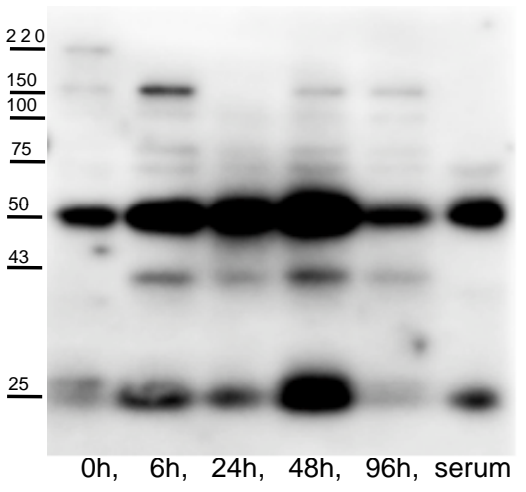

Oh, 6h, 24h, 48h, 96h, serum

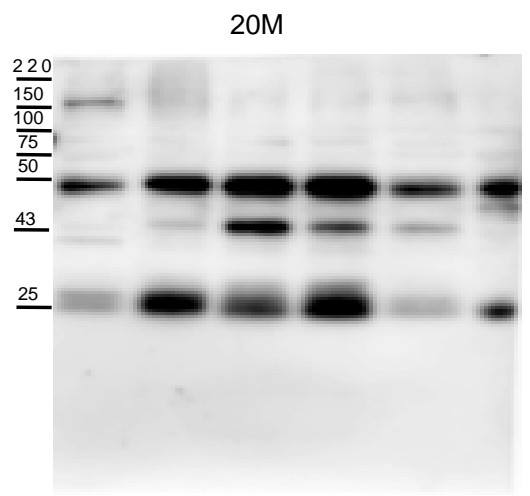

Oh, 6h, 24h, 48h, 96h, serum 

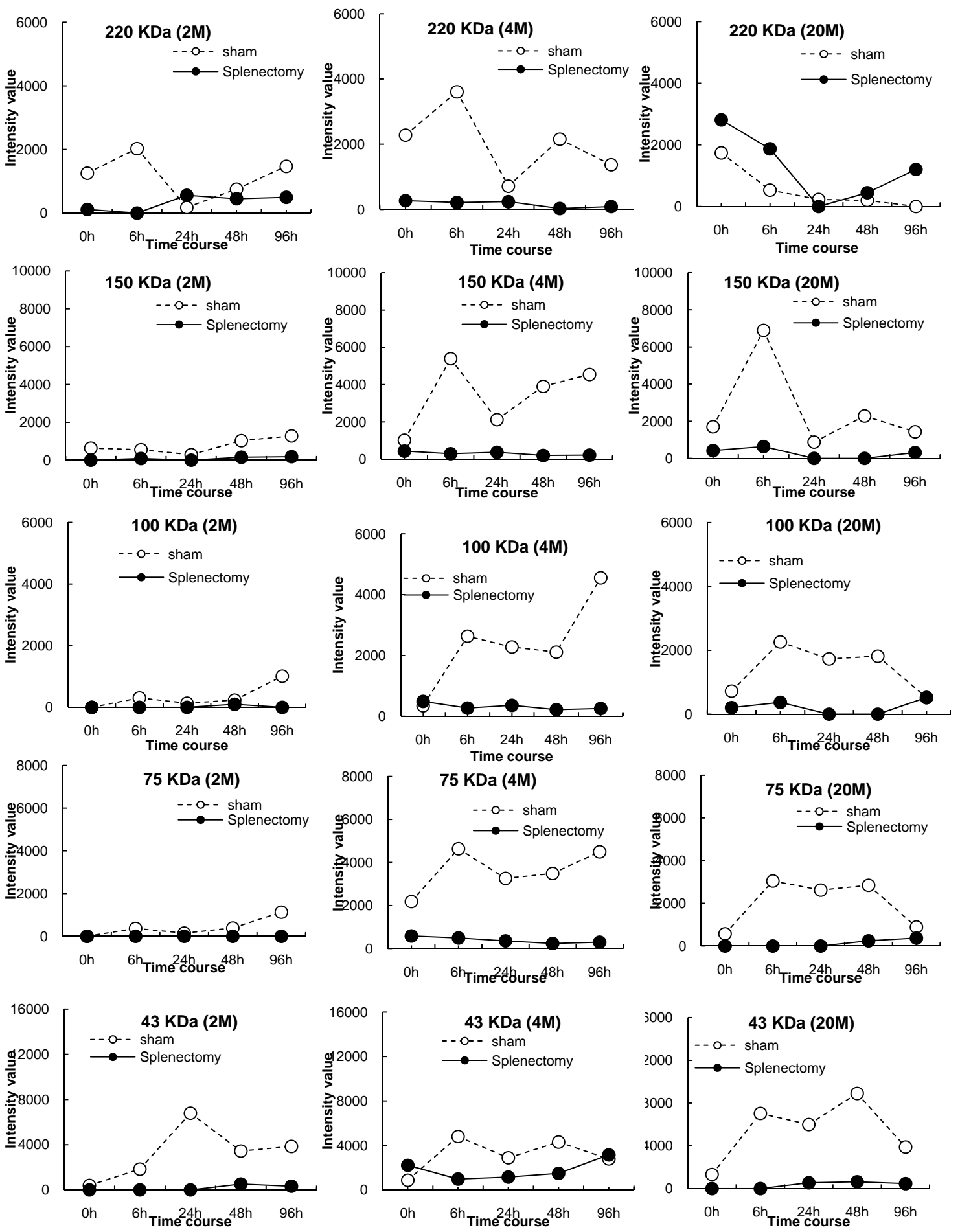

Fig. (3). Age-dependent reduction of IgG1 antibody binding to damaged tissues following splenectomy.

Damaged tissues were taken from 2M old C57BL/6 mice at $6-96 \mathrm{~h}$ after punch biopsy. Tissue lysates/homogenates were subjected to SDS PAGE and transferred to PVDF membranes. Sera were collected from $2 \mathrm{M}, 4 \mathrm{M}$ or $20 \mathrm{M}$ old mice one week after a sham operation or splenectomy. Sera (diluted 1:100) were reacted with membranes as a source of primary antibodies and then membranes were reacted with anti-mouse IgG1 secondary antibodies. A. Bound antibodies were detected by the enhanced chemiluminescence (ECL) system. Representative results are shown for sera taken from five mice at each age. Lines indicate possible bands for quantitative analysis. B. Determination of band intensities by image analysis software. Intensity of each band was determined after subtracting background intensity around the band. 

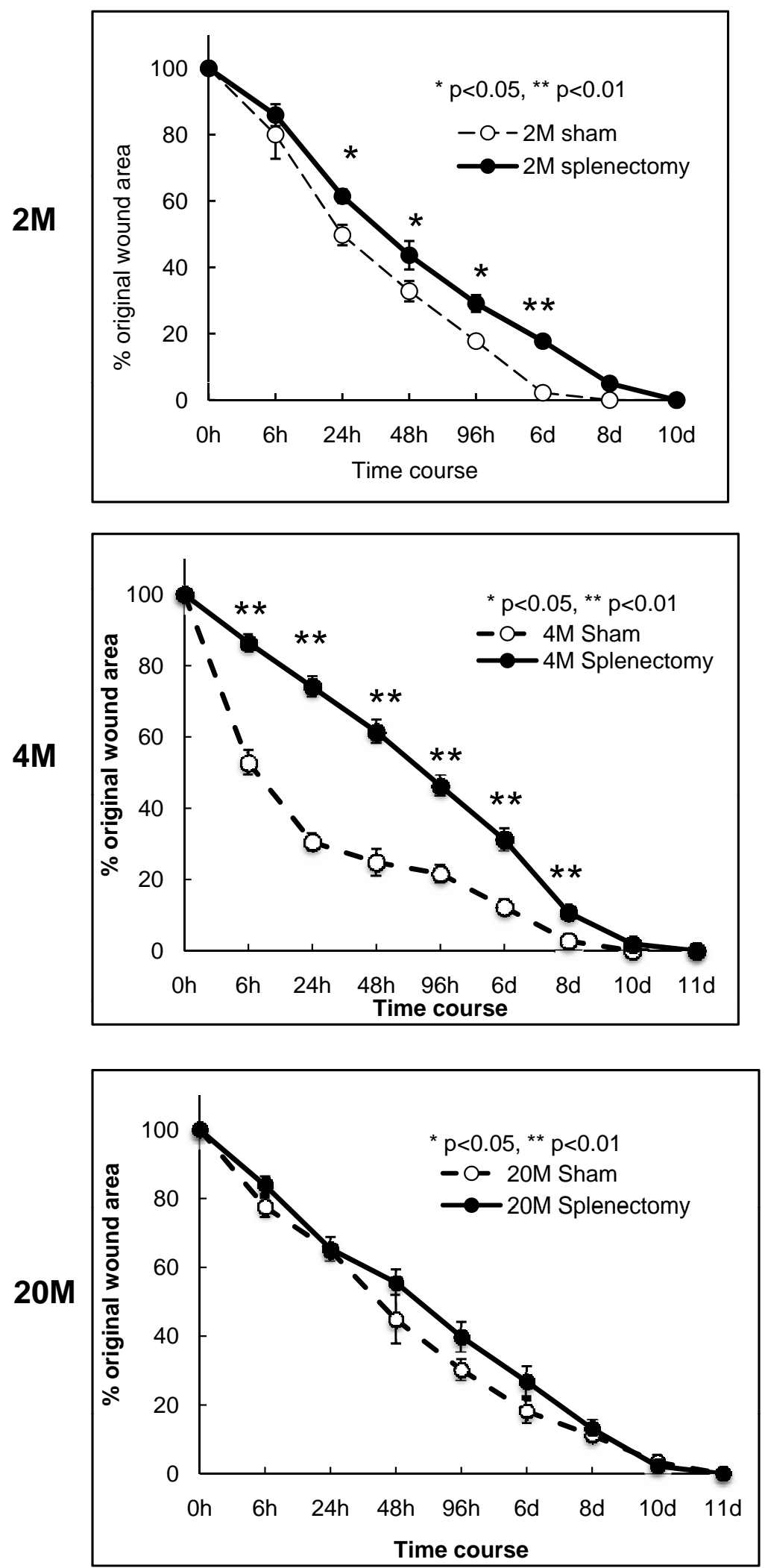

Fig. (4). Delayed wound healing in splenectomized C57BL/6 mice.

Two month, $4 \mathrm{M}$ or $20 \mathrm{M}$ old C57BL/6 mice were splenectomized or subjected to a sham operation. One week later, punch biopsies were performed on the backs of these mice. Changes in the percentages of wound areas are shown at each time point in comparison to the original wound area in splenectomized (closed circles) or sham-operated mice (open circles). Data shown are the mean ratios \pm SEM of five mice. (*; $\mathrm{p}<0.05, * * ; \mathrm{p}<0.01)$. 
(A)
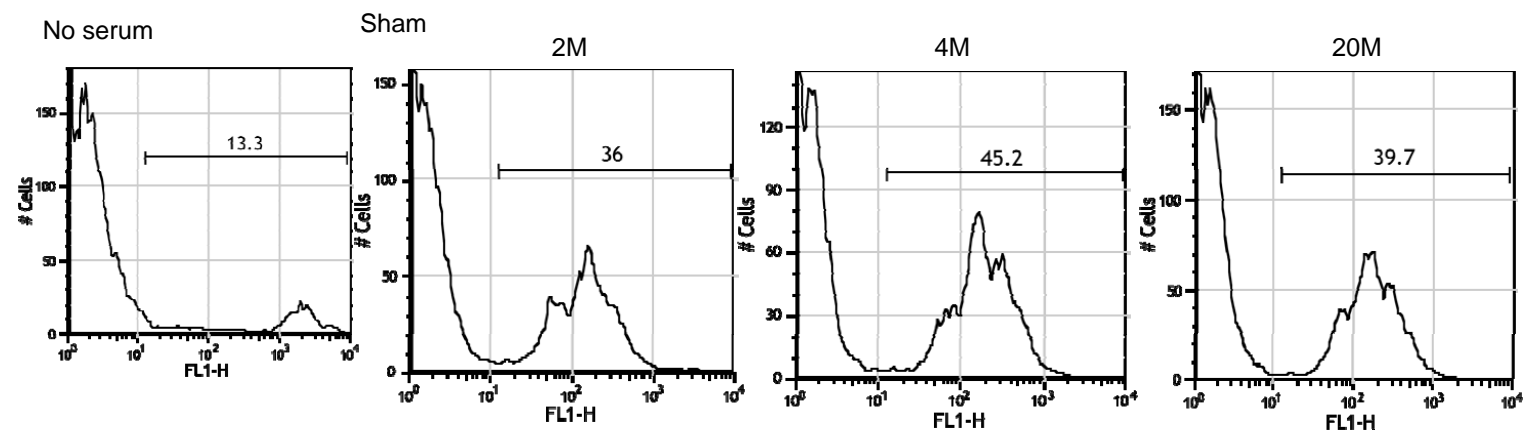

Splenectomy
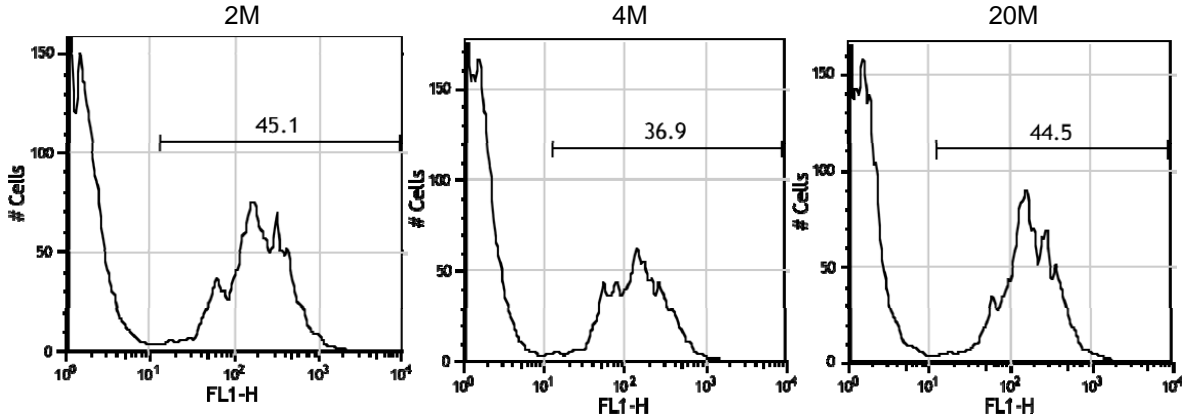

(B)

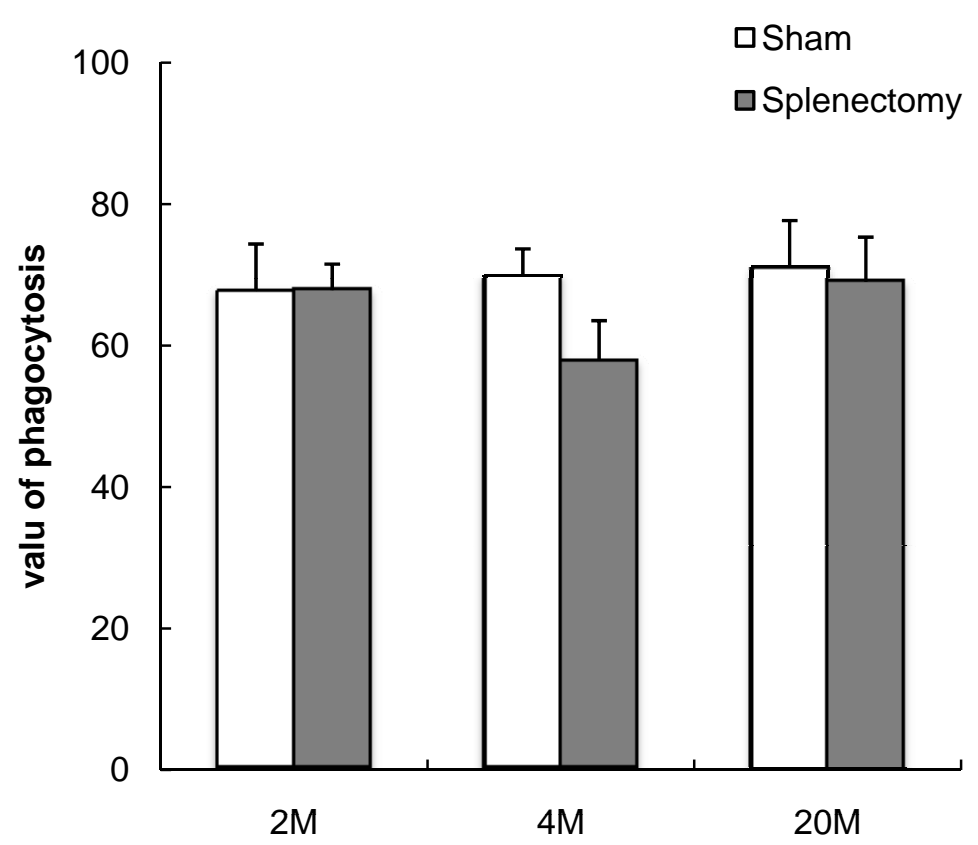

Fig. (5). Serum from aged mice had the same level of opsonizing capacity as that of young mice.

Neutrophils were taken from C57BL/6 as described in Materials and Methods. Serum samples from 2M, 4M or 20M old C57BL/6 mice (sham operated; upper lane, splenectomized; lower lane) were added to heat-treated neutrophils and CFSE-labeled. They were phagocytosed by macrophages taken from C57BL/6 as described in Materials and Methods. A. Representative results of FACS experiments. B. Effects of opsonization. Data were calculated as follows. Value $=(\%$ phagocytosis with serum- $\%$ phagocytosis without serum $) /(\%$ phagocytosis with serum). Data were derived from five experiments using sera taken from five mice at each age.

age. The number and intensity of IgG1 bands bound to wounded tissues at $2 \mathrm{M}$ were lower than those of $4 \mathrm{M}$ old C57BL/6 mice. Although the number and intensity of IgG1 bands bound to wounded tissues increased until $12 \mathrm{M}$ or $17 \mathrm{M}$ of age, those from $20 \mathrm{M}$ old C57BL/6 mice decreased (Fig.
1). Splenectomy strongly reduced IgG1 binding to wounded tissues at $4 \mathrm{M}$ of age. Although the IgG1 bands were reduced by splenectomy at $2 \mathrm{M}$ and $20 \mathrm{M}$ of age, the reduction was less than that observed in $4 \mathrm{M}$ old mice (Fig. 3). These findings correlated with the prominent delay of wound healing 
by splenectomy at $4 \mathrm{M}$ of age. Shorter delays of wound healing following splenectomy were seen in mice at $2 \mathrm{M}$ and $20 \mathrm{M}$ of age (Fig. 4).

We have shown that both IgG1 and IgM antibodies bind to damaged tissues. IgM antibodies to wounded tissues appeared early in life and remained throughout the life of the mice (2 $\mathrm{M}$ to $20 \mathrm{M}$ ) (Fig. 3). IgM, IgG and IgA autoantibodies, reactive with a variety of serum proteins, cell surface structures and intracellular structures, are "naturally" found in all normal individuals (natural autoantibodies, NAAs) [12]. The exact cellular origin of NAAs is unclear. Mature B cells can be divided into two major populations: B1 and B2 cells [13]. B2 cells in the spleen are further divided into two subsets, follicular (FO) and marginal zone (MZ) B cells [14]. Because B1 cell-derived Abs bear similarity to NAAs in that they often recognize self-Ags, it is believed that B1 cells are a major source of NAAs $[13,15,16]$. However, several reports have shown recently the existence of IgG antibodies to auto-antigens. It has been shown that B cells with a low level of self-reactivity or with reactivity to bacterial wall components preferably reside in the MZ [17]. Although B cells are subject to negative selection, B cells can be positively selected, generated and maintained on the basis of their auto-reactivity [18]. Thus, autoreactive, autoantibody-secreting B lymphocytes are found in healthy individuals and are stimulated by auto-antigens. NAAs constitute a large fraction of serum immunoglobulin. Immunoadsorption experiments showed that up to $66 \%$ of IgG from individual sera were auto-reactive $[19,20]$. Because class switching from IgM to IgG occurs in secondary lymph organs such as the spleen, it is reasonable that splenectomy reduces the production of autoreactive $\mathrm{IgG}$ antibody.

Our hypothesis is that IgG1 opsonizes the damaged tissues for subsequent phagocytosis. In order to examine phagocytosis, we used neutrophils as target tissue cells, which were phagocytosed by macrophages. In these experiments, we obtained neutrophils and macrophages from $4 \mathrm{M}$ old mice. Because opsonizing capacity of antibodies is mainly caused by $\operatorname{IgG1}$, the peak reduction of $\mathrm{IgG} 1$ by splenectomy at $4 \mathrm{M}$ may be correlated to the decrease of opsonising capacity (Fig. 5). The reason for similar levels of opsonization from $2 \mathrm{M}$ and $20 \mathrm{M}$ old mouse sera compared to $4 \mathrm{M}$ old mouse serum cannot be explained only by IgG1 binding. Complement, which is activated by $\operatorname{IgM}$ or IgG antibodies (other than IgG1) may also induce opsonization.

Taken together, we have show in this paper that IgG1 opsonizes the damaged tissues, which are removed by phagocytosis by macrophages. This IgG1 dependent opsonization is highest at $4 \mathrm{M}$ old mice, because splenectomy reduces phagocytosis and wound healing only at $4 \mathrm{M}$ old mice.

\section{ACKNOWLEDGEMENT}

We thank Mr. Tanaka in the Division for Medical Research Engineering, Nagoya University Graduate School of Medicine for maintenance of the flow cytometer. This work was supported by the Ministry of Education, Science, Technology, Sports and Culture, Japan.

\section{REFERENCES}

[1] Swift ME, Burn AL, Gray KL, DiPietro LA. Age-related alterations in the inflammatory response to dermal injury. J Invest Dermatol 2001; 117(5): 1027-35.

[2] Singer AJ, Clark RA. Cutaneous wound healing. N Engl J Med 1999; 341(10): 738-46.

[3] Werner S, Grose R. Regulation of wound healing by growth factors and cytokines. Physiol Rev 2003; 83(3): 835-70.

[4] Gillitzer R, Goebeler M. Chemokines in cutaneous wound healing. J Leukoc Biol 2001; 69(4): 513-21.

[5] Martin P, Leibovich SJ. Inflammatory cells during wound repair: the good, the bad and the ugly. Trends Cell Biol 2005; 15: 599-607.

[6] Dovi JV, He LK, Di Pietro LA. Accelerated wound closure in neutrophil-depleted mice. J Leukoc Biol 2003; 73(4): 448-55.

[7] Nishio N, Okawa Y, Sakurai H, Isobe K. Neutrophil depletion delays wound repair in aged mice. AGE 2008; 30: 11-9.

[8] Stuart LM, Ezekowitz RA. Phagocytosis: elegant complexity. Immunity 2005; 22(5): 539-50.

[9] Jameson J, Ugarte K, Chen N, et al. A role for skin T cells in wound repair. Science 2002; 296: 747-9.

[10] Girardi M, Lewis J, Glusac E, et al. Resident skin-specific T cells provide local, nonredundant regulation of cutaneous inflammation. J Exp Med 2002; 195: 855-67.

[11] Nishio N, Ito S, Suzuki H, Isobe K. Antibodies to wounded tissue enhances cutaneous wound healing. Immunology 2009; 128: 369 80 .

[12] Coutinho A, Kazatchkine MD, Avrameas S. Natural autoantibodies. Curr Opin Immunol 1995; 7: 812-8.

[13] Kantor AB, Herzenberg LA. Origin of murine B cell lineages. Annu Rev Immunol 1993; 11: 501-38.

[14] Martin F, Kearney JF. B-cell subsets and the mature preimmune repertoire: marginal zone and B1 B cells as part of a "natural immune memory". Immunol Rev 2000; 175: 70-9.

[15] Hayakawa K, Hardy RR, Parks DR, Herzenberg LA. The "Ly-1": B cell subpopulation in normal, immunodefective, and autoimmune mice. J Exp Med 1983; 157: 202-18.

[16] Stall AM, Wells SM, Lam KP. B1 cells: unique origins and functions. Semin Immunol 1996; 8: 45-59.

[17] Martin F, Kearney JF. Positive selection from newly formed to marginal zone B cells depends on the rate of clonal production, CD19, and btk. Immunity 2000; 12: 39-49.

[18] Hayakawa K, Asano M, Shinton SA, et al. Positive selection of natural autoreactive B cells. Science1999; 285: 113-6.

[19] Avrameas S, Ternynck T. The natural autoantibodies system: between hypotheses and facts. Mol Immunol 1993; 30: 1133-42.

[20] Kohler H, Bayry J, Nicoletti A, Kaveri SV. Natural autoantibodies as tools to predict the outcome of immune response? Scand J Immunol 2003; 58: 285-9.

This is an open access article licensed under the terms of the Creative Commons Attribution Non-Commercial License (http://creativecommons.org/licenses/ by-nc/3.0/) which permits unrestricted, non-commercial use, distribution and reproduction in any medium, provided the work is properly cited. 